Pacific Journal of Mathematics

FEYNMAN INTEGRALS OF NON-FACTORABLE
FINITE-DIMENSIONAL FUNCTIONAL 


\title{
FEYNMAN INTEGRALS OF NON-FACTORABLE FINITE-DIMENSIONAL FUNCTIONALS
}

\author{
G. W. Johnson And D. L. Skoug
}

Let $C_{0}[a, b]$ denote the space of continuous functions $x$ on $[a, b]$ such that $x(a)=0$. Recently Cameron and Storvick defined an operator-valued Feynman integral $J_{q}(F)$ of functionals $F$ on $C_{0}[a, b]$. Let $F(x)=f\left(x\left(t_{1}\right), \cdots, x\left(t_{n}\right)\right)$ where $a=$ $t_{0}<t_{1}<\cdots<t_{n}=b$. The present authors earlier established the existence of $J_{q}(F)$ for functionals $F$ as above under the assumption that $f$ is factorable and bounded. In the present paper it is shown that with the factorability assumption completely removed, $J_{-1}(F)$ may fail to exist even with $f$ required to be in $L_{p}\left(R_{n}\right)$ for $1 \leqq p \leqq \infty$. On the other hand it is shown that $J_{q}(F)$ does exist under the rather surprising condition that $f \in L_{21 \cdots 12}$ where $L_{21 \cdots 12}$ is the set of all complex-valued measurable functions $f$ on $R_{n}(n \geqq 2)$ such that $\|f\|_{21 \cdots 12} \equiv$ $\|f\|<\infty$ where

$$
\begin{aligned}
\|f\| \equiv & \left\{\int _ { - \infty } ^ { \infty } \left[\int_{-\infty}^{\infty}(n-2) \int_{-\infty}^{\infty}\right.\right. \\
& \left.\left.\times\left(\int_{-\infty}^{\infty}\left|f\left(u_{1}, \cdots, u_{n}\right)\right|^{2} d u_{1}\right)^{1 / 2} d u_{2} \cdots d u_{n-1}\right]^{2} d u_{n}\right\}^{1 / 2} .
\end{aligned}
$$

Another positive result shows that if $F$ is an analytic function of a finite sum of factorable functions, then $J_{q}(F)$ exists.

Cameron and Storvick introduced their operator-valued function space integrals and, in particular, their operator-valued Feynman integral in [2]. The study of these integrals has continued in [3, 4,5 , and 6]. A related function space integral associated with a general Gaussian-Markov process instead of the Wiener process has been considered in [1].

Insofar as possible we adopt the definitions and notation of [2 and 4]. Throughout the paper we assume that $F$ has the form given above where $f$ is a measurable function on $R_{n}$.

In Theorem 1, we establish the existence of $I_{\lambda}(F)$ for $\operatorname{Re} \lambda>0$. Here no factorability is required, and the hypotheses on $f$ are minimal enough so that any bounded and some unbounded functions $f$ are allowed. Further we show that $I_{\lambda}(F)$ is the strong operator limit of the operators $I_{\lambda}^{o}(F)$ rather than just the weak operator limit as required by the definition. Comparison of Theorem 1 and the corresponding result in our earlier paper [4, p.777] shows that, even in the factorable setting, the present result is stronger.

Theorems 2 and 3 contain the positive results mentioned above. 
In Theorem 3, the assumptions on $F$ preserve "enough factorability" so that the proof can be carried out. In Theorem 2, the "sufficient condition" requires no factorability. Further work is in progress concerning this condition.

The example mentioned above is perhaps the main contribution of this paper. The functional $F$ in the example is very restricted; $F$ depends on $x$ only through the values of $x$ at three points, and the associated function $f$ on $R_{3}$ is severely restricted, and yet, $J_{-1}(F)$ fails to exist. This example rules out many attractive conjectures in regard to the extent of the existence theory for $J_{q}(F)$.

In the positive results concerning $J_{q}(F)$ the operator $J_{q}(F)$ is obtained as the strong operator limit of the operators $I_{\lambda}(F)$ as $\lambda \rightarrow-i q$ in $C^{+}=\{\lambda \in C: \operatorname{Re} \lambda>0\}$ rather than just the weak operator limit as required by the definition. Further, the existence of $J_{q}(F)$ is obtained for all $q \neq 0$ as in [3, 4, and 6] rather than the indeterminate "almost every nonzero $q$ " as in [2 and 5].

1. Non-factorable finite dimensional functionals. Our first Theorem deals with the existence of the operator $I_{\lambda}(F)$ for $\operatorname{Re} \lambda>0$.

THEOREM 1. Suppose $f\left(u_{1}, \cdots, u_{n}\right)$ is such that for every $p>0$, the function $B_{p}\left(u_{1}, \cdots, u_{n}, \xi\right) \equiv f\left(u_{1}, \cdots, u_{n}\right) \exp \left[-p \sum_{j=1}^{n}\left(u_{j}-u_{j-1}\right)^{2}\right]$ is bounded, say by $H(p)$. Then $I_{\lambda}(F)$ exists for every $\lambda \in C^{+}$and, in fact, $I_{\lambda}^{o}(F) \rightarrow I_{\lambda}(F)$ in the strong operator topology as $\|\sigma\| \rightarrow 0$ (as in earlier papers, $\left.u_{0} \equiv \xi\right)$.

Proof. For $\psi \in L^{2}$ and $\xi \in(-\infty, \infty)$, let

$$
\begin{aligned}
\left(K_{\lambda}(F) \psi\right)(\xi)= & A \lambda^{n / 2} \int_{-\infty}^{\infty}(n) \int_{-\infty}^{\infty} f\left(u_{1}, \cdots, u_{n}\right) \psi\left(u_{n}\right) \\
& \times \exp \left(-\sum_{j=1}^{n} \frac{\lambda\left(u_{j}-u_{j-1}\right)^{2}}{2\left(t_{j}-t_{j-1}\right)}\right) d u_{1} \cdots d u_{n}
\end{aligned}
$$

where $A=\left[(2 \pi)^{n}\left(t_{1}-a\right) \cdots\left(t_{n}-t_{n-1}\right)\right]^{-1 / 2}$.

We wish to show that $K_{\lambda}(F) \in \mathscr{L}\left(L_{2}\right)$. The inequalities necessary to show the boundedness of $K_{\lambda}(F)$ will be given in some detail while similar details will be omitted later on in the proof. First note that

$$
\begin{aligned}
&\left|\left(K_{\lambda}(F) \psi\right)(\xi)\right| \leqq A|\lambda|^{n / 2} \int_{-\infty}^{\infty}(n) \int_{-\infty}^{\infty}\left|f\left(u_{1}, \cdots, u_{n}\right)\right|\left|\psi\left(u_{n}\right)\right| \cdot \\
& \exp \left(-\frac{\operatorname{Re} \lambda}{4(b-a)} \sum_{j=1}^{n}\left(u_{j}-u_{j-1}\right)^{2}\right) \exp \left(-\sum_{j=1}^{n} \frac{\operatorname{Re} \lambda\left(u_{j}-u_{j-1}\right)^{2}}{4\left(t_{j}-t_{j-1}\right)}\right) d u_{1} \cdots d u_{n} \\
& \leqq A H\left(\frac{\operatorname{Re} \lambda}{4(b-a)}\right)|\lambda|^{n / 2} \int_{-\infty}^{\infty}(n) \int_{-\infty}^{\infty} \mid \psi\left(u_{n}\right)
\end{aligned}
$$




$$
\begin{aligned}
& \times \exp \left(-\sum_{j=1}^{n} \frac{\operatorname{Re} \lambda\left(u_{j}-u_{j-1}\right)^{2}}{4\left(t_{j}-t_{j-1}\right)}\right) d u_{1} \cdots d u_{n} \\
= & H\left(\frac{\operatorname{Re} \lambda}{4(b-a)}\right)(2|\lambda| / R e \lambda)^{n / 2}\left(\frac{\operatorname{Re} \lambda}{4 \pi(b-a)}\right)^{1 / 2} \int_{-\infty}^{\infty}\left|\psi\left(u_{n}\right)\right| \\
& \times \exp \left(-\frac{\operatorname{Re} \lambda\left(u_{n}-\xi\right)^{2}}{4(b-a)}\right) d u_{n} .
\end{aligned}
$$

Hence by Lemma 1 of [2],

$$
\left\|K_{\lambda}(F) \psi\right\| \leqq H\left(\frac{\operatorname{Re} \lambda}{4(b-a)}\right)(2|\lambda| / \operatorname{Re} \lambda)^{n / 2}\|\psi\|
$$

and $K_{\lambda}(F) \in \mathscr{L}\left(\mathrm{L}_{2}\right)$.

Now the argument given in [2, p.530] shows that for $\lambda>0, K_{\lambda}(F)=$ $I_{\lambda}(F)$. Further we claim that an application of Morera's theorem shows that $K_{\lambda}(F)$ is an operator-valued analytic function of $\lambda$ for $\lambda \in C^{+}$. To establish this, it suffices to show that for every $\psi, \phi \in$ $L_{2}, h(\lambda)=\left(K_{\lambda}(F) \psi, \phi\right)$ is an analytic function of $\lambda$ in $C^{+}$. To begin with one shows that $h$ is continuous in $C^{+}$. Let $\lambda \in C^{+}$and suppose that $\left\{\lambda_{i}\right\}$ is a sequence in $C^{+}$such that $\lambda_{i} \rightarrow \lambda$. Let $D$ be an upper bound for $\left\{\left|\lambda_{i}\right|\right\}$ and $E>0$ be a lower bound for $\left\{\operatorname{Re} \lambda_{i}\right\}$. To obtain the continuity, one applies the Dominated Convergence Theorem. The expression

$$
D^{n / 2} A H\left(\frac{E}{4(b-a)}\right)\left|\psi\left(u_{n}\right)\right||\phi(\xi)| \exp \left(-\sum_{j=1}^{n} \frac{E\left(u_{j}-u_{j-1}\right)^{2}}{4\left(t_{j}-t_{j-1}\right)}\right)
$$

will serve as a dominating function and is an integrable function of $u_{1}, \cdots, u_{n}$, and $\xi$.

Now let $\Gamma$ be a triangular path in $C^{+}$. We wish to show $\int_{\Gamma}\left(K_{\lambda}(F) \psi, \phi\right) d \lambda=0$. This is clear from the Cauchy Integral Theorem if one can justify moving the integral with respect to $\lambda$ inside the other integrals. But, if $D$ is chosen as an upper bound for $\{|\lambda|: \lambda \in \Gamma\}$ and $E>0$ is chosen as a lower bound of $\{\operatorname{Re} \lambda: \lambda \in \Gamma\}$ then the function in (2) is integrable with respect to $u_{1}, \cdots, u_{n}, \xi$, and $\lambda$, and dominates the function in question. Hence the use of Fubini's theorem is justified.

Since $K_{\lambda}(F)$ is analytic in $C^{+}$and agrees with $I_{\lambda}(F)$ for $\lambda>0$, $I_{\lambda}^{a n}(F)$ exists throughout $C^{+}$and equals $K_{\lambda}(F)$.

We finish the proof by showing that $I_{\lambda}^{o}(F) \rightarrow I_{\lambda}(F)$ in the strong operator topology as $\|\sigma\| \rightarrow 0$ for each fixed $\lambda \in C^{+}$. It suffices to consider a fixed $\psi \in L_{2}$ and a sequence $\left\{\sigma_{i}\right\}$ of partitions such that $\left\|\sigma_{i}\right\| \rightarrow 0$ and show that $\left\|I_{\lambda}^{\sigma i}(F) \psi-K_{\lambda}(F) \psi\right\| \rightarrow 0$. We will do this by showing (i) there exists an $L_{2}$-function which dominates $I_{i}^{\sigma}(F)$ for $\left\|\sigma_{i}\right\|$ suf- 
ficiently small and (ii) $\left(I_{\lambda}^{\sigma_{i}}(F) \psi\right)(\xi) \rightarrow\left(K_{\lambda}(F) \psi\right)(\xi)$ for all $\xi \in(-\infty, \infty)$. (i) implies that the sequence $\left\{\left\|I_{\lambda}^{\sigma_{i}}(F) \psi\right\|\right\}$ is bounded, and that, along with (ii) implies that $I_{\lambda}^{\sigma_{i}}(F) \psi \rightarrow K_{\lambda}(F) \psi$ weakly. (i) and (ii) allow one to use the Dominated Convergence Theorem and write

$$
\left\|I_{\Lambda}^{\sigma_{i}}(F) \psi\right\|^{2}=\int_{-\infty}^{\infty}\left|\left(I_{\lambda}^{\sigma_{i}}(F) \psi\right)(\xi)\right|^{2} d \xi \longrightarrow \int_{-\infty}^{\infty}\left|\left(K_{\lambda}(F) \psi\right)(\xi)\right|^{2} d \xi=\left\|K_{\lambda}(F) \psi\right\|^{2}
$$

and this, along with weak convergence, implies the desired strong convergence.

So it remains to establish (i) and (ii). For the rest of the proof we consider partitions $\sigma_{i}: a=s_{0}<s_{1}<\cdots<s_{m}=b$ such that $2\left\|\sigma_{i}\right\|<$ $\min \left\{t_{1}-a, \cdots, t_{n}-t_{n-1}\right\}$. By carrying out $m-n$ integrations we obtain

$$
\begin{aligned}
\left(I_{\lambda}^{\sigma_{i}}(F) \psi\right)(\xi)= & \lambda^{n / 2}\left[(2 \pi)^{n}\left(r_{1}-a\right) \cdots\left(r_{n}-r_{n-1}\right)\right]^{-1 / 2} \int_{-\infty}^{\infty}(n) \int_{-\infty}^{\infty} f\left(u_{1}, \cdots, u_{n}\right) \\
& \times \psi\left(u_{n}\right) \exp \left(-\sum_{j=1}^{n} \frac{\lambda\left(u_{j}-u_{j-1}\right)^{2}}{2\left(r_{j}-r_{j-1}\right)}\right) d u_{1} \cdots d u_{n}
\end{aligned}
$$

where $r_{0}=a$ and $r_{j}$ is that $s_{K}$ such that $s_{K} \leqq t_{j}<s_{K+1}$. Hence

$$
\begin{aligned}
& \left|\left(I_{\lambda}^{\sigma_{i}}(F) \psi\right)(\xi)\right| \\
& \leqq \\
& \qquad H\left(\frac{\operatorname{Re} \lambda}{4 \pi(b-a)}\right)(2 \lambda)^{n / 2} \int_{-\infty}^{\infty}(n) \int_{-\infty}^{\infty}\left|\psi\left(u_{n}\right)\right| \\
& \quad \times \exp \left(-\sum_{j=1}^{n} \frac{\operatorname{Re} \lambda\left(u_{j}-u_{j-1}\right)^{2}}{4(b-a)}\right) d u_{1} \cdots d u_{n} \\
& =(\text { constant })\left(\frac{\operatorname{Re} \lambda}{4 \pi n(b-a)}\right)^{1 / 2} \int_{-\infty}^{\infty}\left|\psi\left(u_{n}\right)\right| \exp \left(-\frac{\operatorname{Re} \lambda\left(u_{n}-\xi\right)^{2}}{4 n(b-a)}\right) d u_{n} .
\end{aligned}
$$

Now by Lemma 1 of [2], the right hand side of 3 is an $L_{2}$-function of $\xi$, which establishes (i). To obtain (ii), fix $\xi$ and note that since the integrand in the right hand side of (3) is the product of two $L_{2}$ functions, it is integrable. Hence the integrand in the middle term of (3) is a dominating integrable function and since $r_{j} \rightarrow t_{j}$ as $\left\|\sigma_{i}\right\| \rightarrow 0$, the Dominated Convergence Theorem gives the desired pointwise convergence.

In the next theorem we show that if $f \in L_{21 \cdots 12}$ then the operator $J_{q}(F)$ exists. On the other hand we show in an example below that if the condition $f \in L_{21 \cdots 12}$ is replaced by the condition $f \in L_{p}\left(R_{n}\right)$ for $1 \leqq p \leqq \infty$, then the operator $J_{q}(F)$ need not exist. We mention that $\left(L_{21 \cdots 12},\|\cdot\|_{21 \cdots 12}\right)$ is a Banach space; this can be shown in much the same way that one shows $L_{p}$ to be a Banach space.

THEOREM 2. Let $F(x)=f\left(x\left(t_{1}\right), \cdots, x\left(t_{n}\right)\right), n \geqq 2$, where $f \in L_{21 \cdots 12}$ • Then $I_{\lambda}(F)$ exists for every $\lambda \in C^{+}$and is given by (1). Furthermore 
$J_{q}(F)$ exists for all real $q \neq 0$ and $I_{\lambda}(F) \rightarrow J_{q}(F)$ in the strong operator topology as $\lambda \rightarrow-i q$. Also $J_{q}(F)$ is given by

$$
\begin{aligned}
\left(J_{q}(F) \psi\right)(\xi)= & \left(\frac{q}{2 \pi i\left(t_{1}-a\right)}\right)^{1 / 2(\xi)} \int_{-\infty}^{\infty} \\
& \times \exp \left(\frac{q i\left(u_{1}-\xi\right)^{2}}{2\left(t_{1}-a\right)}\right) g\left(\psi ;-q i, u_{1}\right) d u_{1}
\end{aligned}
$$

and

$$
\left\|J_{q}(F)\right\| \leqq p(|q|)\|f\|
$$

where

$$
p(\lambda)=(\lambda / 2 \pi)^{(n-1) / 2}\left[\left(t_{2}-t_{1}\right) \cdots\left(t_{n}-t_{n-1}\right)\right]^{-1 / 2}
$$

and

$$
\begin{aligned}
g\left(\psi ; \lambda, u_{1}\right)= & p(\lambda) \int_{-\infty}^{\infty}(n-1) \int_{-\infty}^{\infty} f\left(u_{1}, \cdots, u_{n}\right) \psi\left(u_{n}\right) \\
& \times \exp \left[-\sum_{j=2}^{n} \frac{\lambda\left(u_{j}-u_{j-1}\right)^{2}}{2\left(t_{j}-t_{j-1}\right)}\right] d u_{2} \cdots d u_{n}
\end{aligned}
$$

for $\psi \in L_{2}$ and $R e \lambda \geqq 0, \lambda \neq 0$.

Proof. First we see that for $\psi \in L_{2}, g(\psi ; \lambda, \cdot)$ is in $L_{2}$ for all $\lambda \neq 0$ such that $\operatorname{Re} \lambda \geqq 0$. This follows since, by the Schwarz inequality, we have

$$
\begin{aligned}
& \|g(\psi ; \lambda, \cdot)\|^{2} \\
& =\int_{-\infty}^{\infty}\left|g\left(\psi ; \lambda, u_{1}\right)\right|^{2} d u_{1} \leqq p^{2}(|\lambda|) \int_{-\infty}^{\infty}(2 n-2) \int_{-\infty}^{\infty}\left|\psi\left(u_{n}\right)\right|\left|\psi\left(w_{n}\right)\right| \\
& \times\left[\int_{-\infty}^{\infty}\left|f\left(u_{1}, u_{2}, \cdots, u_{n}\right)\right|\left|f\left(u_{1}, w_{2}, \cdots, w_{n}\right)\right| d u_{1}\right] d u_{2} \cdots d u_{n} d w_{2} \cdots d w_{n} \\
& \leqq\left(p(|\lambda|) \int_{-\infty}^{\infty}\left|\psi\left(u_{n}\right)\right|\left[\int_{-\infty}^{\infty}(n-2) \int_{-\infty}^{\infty}\left\|f\left(\cdot, u_{2}, \cdots, u_{n}\right)\right\| d u_{2} \cdots d u_{n-1}\right] d u_{n}\right)^{2} \\
& =\left\{p ( | \lambda | ) \int _ { - \infty } ^ { \infty } | \psi ( u _ { n } ) | \left[\int_{-\infty}^{\infty}(n-2) \int_{-\infty}^{\infty}\left(\int_{-\infty}^{\infty}\left|f\left(u_{1}, \cdots, u_{n}\right)\right|^{2} d u_{1}\right)^{1 / 2}\right.\right. \\
& \left.\left.\times d u_{2} \cdots d u_{n-1}\right] d u_{n}\right\}^{2} \leqq[p(|\lambda|)\|\psi\|\|f\|]^{2}<\infty .
\end{aligned}
$$

Thus the right side of (1) and the right side of (4) define operators, which we shall temporarily denote by $K_{\lambda}(F)$ and $K_{q}(F)$ respectively. By Lemma 1 of [2] we have

$$
\left\|K_{\lambda}(F)\right\| \leqq p(|\lambda|)\|f\| \text { and }\left\|K_{q}(F)\right\| \leqq p(|q|)\|f\| \text {. }
$$

Next we wish to show that $I_{\lambda}(F)$ exists for all $\lambda \in C^{+}$and is given by (1). First we note that for $\lambda>0, I_{\lambda}(F)$ exists and equals $K_{\lambda}(F)$ (In fact that is how $K_{\lambda}(F)$ was obtained; we evaluated the 
Wiener integral defining $I_{\lambda}(F)$ for $\lambda>0$, took the obtained expression and used it to define $K_{\lambda}(F)$ for $\lambda \in C^{+}$.). We need to show that $I_{\lambda}^{\text {seq }}(F)$ and $I_{\lambda}^{a n}(F)$ both exist and equal $K_{\lambda}(F)$. We claim that an application of Morera's Theorem shows that $K_{\lambda}(F)$ is an operatorvalued analytic function of $\lambda$ in $C^{+}$. The details are similar to those given in the proof of Theorem 1 above. Thus $I_{\lambda}^{a n}(F)$ exists and equals $K_{\lambda}(F)$ throughout $C^{+}$. Next we will show that $I_{\lambda}^{\sigma}(F) \rightarrow K_{\lambda}\left(F^{\prime}\right)$ in the strong operator topology as $\|\sigma\| \rightarrow 0$ for each fixed $\lambda \in C^{+}$. Again, as in the proof of Theorem 1, it suffices to consider a fixed $\psi \in L_{2}$ and a sequence $\left\{\sigma_{i}\right\}$ of partitions such that $\left\|\sigma_{i}\right\| \rightarrow 0$ and show that; (i) there exists an $L_{2}$-function, say $G(\xi)$, which dominates $\left(I_{i}^{\sigma_{i}}(F) \psi\right)(\xi)$ for $\left\|\sigma_{i}\right\|$ sufficiently small, and (ii) $\left(I_{\lambda}^{\sigma_{i}}(F) \psi\right)(\xi) \rightarrow\left(K_{\lambda}(F) \psi\right)(\xi)$ as $\left\|\sigma_{i}\right\| \rightarrow 0$ for almost all real $\xi$. To establish (i), note that for any partition $\sigma_{i}: a=s_{0}<s_{1}<\cdots<s_{m}=b$ such that $2\left\|\sigma_{i}\right\|<\min$ $\times\left\{t_{1}-a, \cdots, t_{n}-t_{n-1}\right\}=d$ we have (see (3) and the preceding equation) that $\left(I_{\dot{\alpha}}^{\sigma i}(F) \psi\right)(\xi)$ is dominated by $G(\xi)$ where $G$ is defined by

$$
\begin{aligned}
G(\xi)= & (|\lambda| / \pi d)^{n / 2}\left(\frac{2 \pi(b-a)}{\operatorname{Re} \lambda}\right)^{1 / 2}\left(\frac{\operatorname{Re} \lambda}{2 \pi(b-a)}\right)^{1 / 2} \int_{-\infty}^{\infty} \exp \left(\frac{-\operatorname{Re} \lambda\left(u_{1}-\xi\right)^{2}}{2(b-a)}\right) \\
& \times\left[\int_{-\infty}^{\infty}(n-1) \int_{-\infty}^{\infty}\left|\psi\left(u_{n}\right) f\left(u_{1}, \cdots, u_{n}\right)\right| d u_{2} \cdots d u_{n}\right] d u_{1} .
\end{aligned}
$$

Since $\int_{-\infty}^{\infty}(n-1) \int_{-\infty}^{\infty}\left|\psi\left(u_{n}\right) f\left(u_{1}, \cdots, u_{n}\right)\right| d u_{2} \cdots d u_{n}$ is an $L_{2}$-function of $u_{1}, G$ is in $L_{2}$. Moreover $G(\xi)$ is always finite since the exponential is also an $L_{2}$-function of $u_{1}$. To establish (ii), let $\xi$ be any fixed real number. Then for any partition $\sigma_{i}: a=s_{0}<s_{1}<\cdots<s_{m}=b$ such that $2\left\|\sigma_{i}\right\|<d$ we have (see the equation and notation preceding (3)) that the quantity

$$
\begin{gathered}
\lambda^{n / 2}\left[(2 \pi)^{n}\left(r_{1}-a\right) \cdots\left(r_{n}-r_{n-1}\right)\right]^{-1 / 2} \psi\left(u_{n}\right) f\left(u_{1}, \cdots, u_{n}\right) \\
\times \exp \left[-\sum_{j=1}^{n} \frac{\lambda\left(u_{j}-u_{j-1}\right)^{2}}{2\left(r_{j}-r_{j-1}\right)}\right]
\end{gathered}
$$

is dominated by the quantity

$$
\left(\frac{|\lambda|}{\pi d}\right)^{n / 2}\left|\psi\left(u_{n}\right) f\left(u_{1}, \cdots, u_{n}\right)\right| \exp \left[-\sum_{j=1}^{n} \frac{\operatorname{Re} \lambda\left(u_{j}-u_{j-1}\right)^{2}}{2(b-a)}\right]
$$

which is in $L_{1}\left(R_{n}\right)$ as a function of $u_{1}, \cdots, u_{n}$. Thus by the Dominated Convergence Theorem

$$
\begin{aligned}
\lim _{\left\|\sigma_{i}\right\| \rightarrow 0} & \left(I_{\lambda}^{\sigma_{i}}(F) \psi\right)(\xi) \\
= & \lim _{\left\|\sigma_{i}\right\| \rightarrow 0} \lambda^{n / 2}\left[(2 \pi)^{n}\left(r_{1}-a\right) \cdots\left(r_{n}-r_{n-1}\right)\right]^{-1 / 2} \int_{-\infty}^{\infty}(n) \int_{-\infty}^{\infty} \psi\left(u_{n}\right) \\
& \times f\left(u_{1}, \cdots, u_{n}\right) \exp \left[-\sum_{j=1}^{n} \frac{\lambda\left(u_{j}-u_{j-1}\right)^{2}}{2\left(r_{j}-r_{j-1}\right)}\right] d u_{1} \cdots d u_{n}
\end{aligned}
$$




$$
\begin{aligned}
= & \lambda^{n / 2}\left[(2 \pi)^{n}\left(t_{1}-a\right) \cdots\left(t_{n}-t_{n-1}\right)\right]^{-1 / 2} \int_{-\infty}^{\infty}(n) \int_{-\infty}^{\infty} \psi\left(u_{n}\right) \\
& \times f\left(u_{1}, \cdots, u_{n}\right) \exp \left[-\sum_{j=1}^{n} \frac{\lambda\left(u_{j}-u_{j-1}\right)^{2}}{2\left(t_{j}-t_{j-1}\right)}\right] d u_{1} \cdots d u_{n} \\
= & \left(K_{\lambda}(F) \psi\right)(\xi) .
\end{aligned}
$$

Thus $I_{\lambda}^{s e q}(F)$ exists and equals $K_{\lambda}(F)$ throughout $C^{+}$.

We will finish the proof by showing that $I_{\lambda}(F) \rightarrow K_{q}(F)$ in the strong operator topology as $\lambda \rightarrow-i q$.

For $g \in L_{2}$ let $\left(C_{2} g\right)(\xi)$

$$
=\left(\lambda / 2 \pi\left(t_{1}-a\right)\right)^{1 / 2} \int_{-\infty}^{\infty} \exp \left(\frac{-\lambda(u-\xi)^{2}}{2\left(t_{1}-a\right)}\right) g(u) d u
$$

and

$$
\left(U_{q} g\right)(\xi)=\left(q / 2 \pi i\left(t_{1}-a\right)\right)^{1 / 2(\xi)} \int_{-\infty}^{\infty} \exp \left(\frac{q i(u-\xi)^{2}}{2\left(t_{1}-a\right)}\right) g(u) d u .
$$

Let $\left\{\lambda_{m}\right\}$ be a sequence in $C^{+}$such that $\lambda_{m} \rightarrow-i q$ as $m \rightarrow \infty$ and such that $\left|\lambda_{m}\right|<|q|+1$ for all $m$. We need to show that $\left\|I_{\lambda_{m}}(F) \psi-K_{q}(F) \psi\right\| \rightarrow 0$ as $m \rightarrow \infty$. Clearly it suffices to do this for $\psi$ a continuous function with compact support.

Note that

$$
\begin{aligned}
\left\|I_{\lambda_{m}}(F) \psi-K_{q}(F) \psi\right\|= & \left\|C_{\lambda_{m}} g\left(\psi ; \lambda_{m}, \cdot\right)-U_{q} g(\psi ;-i q, \cdot)\right\| \\
\leqq & \left\|C_{\lambda_{m}}\right\|\left\|g\left(\psi ; \lambda_{m}, \cdot\right)-g(\psi ;-i q, \cdot)\right\| \\
& +\left\|C_{\lambda_{m}} g(\psi ;-i q, \cdot)-U_{q} g(\psi ;-i q, \cdot)\right\| .
\end{aligned}
$$

But in [4, pp. 776-779] we showed that $\left\|C_{\lambda_{m}}\right\|=1$ and that $\| C_{\lambda_{m}} g(\psi ;-$ $i q, \cdot)-U_{q} g(\psi ;-i q, \cdot) \| \rightarrow 0$ as $m \rightarrow \infty$. Hence it suffices to show $\left\|g\left(\psi ; \lambda_{m}, \cdot\right)-g(\psi ;-i q, \cdot)\right\| \rightarrow 0$ as $m \rightarrow \infty$. This follows by use of the Dominated Convergence Theorem since $g\left(\psi ; \lambda_{m}, u_{1}\right)$ is dominated by the $L_{2}$-function $G_{1}$ defined by

$$
G_{1}\left(u_{1}\right)=p(1+|q|) \int_{-\infty}^{\infty}(n-1) \int_{-\infty}^{\infty}\left|f\left(u_{1}, \cdots, u_{n}\right)\right|\left|\psi\left(u_{n}\right)\right| d u_{2} \cdots d u_{n}
$$

while $g\left(\psi ; \lambda_{m}, u_{1}\right) \rightarrow g\left(\psi ;-i q, u_{1}\right)$ as $m \rightarrow \infty$ for all $u_{1}$ such that $G_{1}\left(u_{1}\right)$ is finite.

COROLlary. Let $F(x)$ be as in Theorem 2 and assume that $f\left(u_{1}, \cdots, u_{n}\right)=\prod_{j=1}^{n} f_{j}\left(u_{j}\right)$ where $f_{1}, f_{n} \in L_{2}(-\infty, \infty)$ and $f_{j} \in L_{1}(-\infty, \infty)$ for $j=2,3, \cdots, n-1$. Then the conclusions of Theorem 2 hold.

Note that even in the factorable case, this corollary yields the 
existence of $J_{q}(F)$ for some $F$ 's not covered by the earlier Theorem $[4, \mathrm{p} .778]$ as the $f_{j}$ 's above need not be bounded.

Next we come to the example which was discussed in some detail in the introduction. In addition to our earlier remarks, note that in case $n=2, L_{2}\left(R_{2}\right)=L_{22}$, so that in order to obtain an example of the type we have, one must go at least to a function $f$ on $R_{3} ; R_{2}$ is not enough.

EXAMPLE. For convenience let $a=0, t_{1}=1, t_{2}=2, t_{3}=3=b$. Let

$$
f(x, y, z)=(\ln z)^{-2}(\ln y)^{-2} \exp \left[-x y z / 2+i(z-y)^{2} / 2+i(y-x)^{2} / 2+i x^{2} / 2\right]
$$

for $x>0, y \geqq 2, z \geqq 2$ and let $f$ vanish elsewhere. Clearly $f$ is bounded by $(\ln 2)^{-4}$ and so $f \in L_{p}\left(R_{3}\right)$ for $1 \leqq p \leqq \infty$ since

$$
\int_{-\infty}^{\infty} \int_{-\infty}^{\infty} \int_{-\infty}^{\infty}|f(x, y, z)| d x d y d z=2(\ln 2)^{-2} \text {. }
$$

Let $F(x)=f(x(1), x(2), x(3))$. We will show that $J_{-1}(F)$ doesn't exist by finding $\phi \in L_{2}$ such that $\lim _{\lambda \rightarrow i} I_{\lambda}(F) \phi$ doesn't exist in the weak topology on $L_{2}$. As a matter of fact, $H(\xi) \equiv \lim _{\lambda \rightarrow i}\left(I_{\lambda}(F) \phi\right)(\xi)$ will exist for all $\xi \in(-\infty, \infty)$ and $H$ will be a bounded uniformly continuous function, but $H \notin L_{2}$.

By Theorem 1 we know that $I_{\lambda}(F)$ exists for all $\lambda \in C^{+}$and is given by the right side of (1). For $\psi \in L_{2}$ let (as in the proof of Theorem 2, we use $\left(K_{q}(F) \psi\right)(\xi)$ to denote the right hand side of equations (1) and (4) when $\lambda=-q i, q$ real and nonzero)

$$
\left(K_{-1}(F) \psi\right)(\xi)=(i / 2 \pi)^{1 / 2} e^{-i \xi^{2} / 2} \int_{-\infty}^{\infty} e^{i \xi x} g(\psi ; x) d x
$$

where

$$
g(\psi ; x)= \begin{cases}(i / 2 \pi) \int_{2}^{\infty} \int_{2}^{\infty}(\ln z)^{-2}(\ln y)^{-2} \exp (-x y z / 2) \psi(z) d y d z, & x>0 \\ 0 & , \quad x \leqq 0 .\end{cases}
$$

We will establish the following; (a) For each $\psi \in L_{2},\left(I_{\lambda}(F) \psi\right)(\xi) \rightarrow$ $\left(K_{-1}(F) \psi\right)(\xi)$ as $\lambda \rightarrow i$ for all $\xi \in(-\infty, \infty)$, (b) there exists $\phi \in L_{2}$ such that $K_{-1}(F) \phi \notin L_{2}$, and (c) $I_{\lambda}(F) \phi$ doesn't converge weakly to an $L_{2}$ function as $\lambda \rightarrow i$ (and hence $J_{-1}\left(F^{\prime}\right)$ doesn't exist).

First we note that $g(\psi ; x)$ exists pointwise in $x$ for all $\psi \in L_{2}$ since for $x>0$,

$$
|g(\psi ; x)| \leqq\left[\pi x(\ln 2)^{2}\right]^{-1} \int_{2}^{\infty} \frac{|\psi(z)| d z}{z(\ln z)^{2}}<\infty .
$$

Furthermore, $g(\psi ; \cdot) \in L_{1}(-\infty, \infty)$ for all $\psi \in L_{2}$ since 


$$
\int_{-\infty}^{\infty}|g(\psi ; x)| d x \leqq 2 \int_{2}^{\infty} \frac{|\psi(z)|}{z(\ln z)^{2}} d z \int_{2}^{\infty} \frac{d y}{y(\ln y)^{2}} .
$$

Thus (a) follows upon use of the Dominated Convergence Theorem.

Now let $\phi(z)=z^{-1 / 2}(\ln z)^{-1}$ for $z \geqq 2$ and let $\phi(z)$ vanish for $z<2$. Clearly $\phi \in L_{2}, \phi \notin L_{1}$ and $\phi(z) \geqq 0$ for all $z$. We claim that $g(\phi ; \cdot) \notin L_{2}$. This follows since

$$
\begin{aligned}
\| & g(\phi ; \cdot) \|^{2}=\int_{0}^{\infty}|g(\phi ; x)|^{2} d x \\
= & (2 \pi)^{-2} \int_{0}^{\infty}\left[\int_{2}^{\infty} \int_{2}^{\infty}(\ln z)^{-2}(\ln y)^{-2} e^{-(x y z) / 2} \phi(z) d y d z\right]^{2} d x \\
= & (2 \pi)^{-2} \int_{2}^{\infty} \phi(z)(\ln z)^{-2} \int_{2}^{\infty} \phi(t)(\ln t)^{-2} \int_{2}^{\infty}(\ln y)^{-2} \int_{2}^{\infty}(\ln s)^{-2} \\
& \times \int_{0}^{\infty} \exp (-x(y z+s t) / 2) d x d s d y d t d z \\
= & 2(2 \pi)^{-2} \int_{2}^{\infty} \phi(z)(\ln z)^{-2} \int_{2}^{\infty} \phi(t)(\ln t)^{-2} \int_{2}^{\infty}(\ln y)^{-2} \int_{2}^{\infty}(\ln s)^{-2} \\
& \times(s t+y z)^{-1} d s d y d t d z \\
\geqq & 2(2 \pi)^{-2} \int_{2}^{\infty} \phi(z)(\ln z)^{-2} \int_{2}^{\infty} \phi(t)(\ln t)^{-2} \int_{t}^{\infty}(\ln y)^{-2} \int_{z}^{\infty}(\ln s)^{-2} \\
& \times(s t+y z)^{-1} d s d y d t d z \\
\geqq & (2 \pi)^{-2} \int_{2}^{\infty} \phi(z)(\ln z)^{-2} \int_{2}^{\infty} \phi(t)(\ln t)^{-2} \int_{t}^{\infty} y^{-1}(\ln y)^{-2} \int_{z}^{\infty} s^{-1}(\ln s)^{-2} d s d y d t d z \\
= & (2 \pi)^{-2} \int_{2}^{\infty} \phi(z)(\ln z)^{-3} d z \int_{2}^{\infty} \phi(t)(\ln t)^{-3} d t \\
= & (2 \pi)^{-2} \int_{2}^{\infty} z^{-1 / 2}(\ln z)^{-4} d z \int_{2}^{\infty} t^{-1 / 2}(\ln t)^{-4} d t=+\infty \cdot
\end{aligned}
$$

But $g(\phi ; \cdot) \notin L_{2}$ implies $K_{-1}(F) \phi \notin L_{2}$ by the Plancherel theorem. Thus (b) is established.

To establish (c), assume $I_{\lambda}(F) \phi$ converges weakly to an $L_{2}$-function as $\lambda \rightarrow i$. Let $\left\{\lambda_{m}\right\}$ be a sequence in $C^{+}$such that $\lambda_{m} \rightarrow i$ as $m \rightarrow \infty$. As $\left\{I_{\lambda_{m}}(F) \phi\right\}$ converges weakly there exists $M \geqq 0$ such that $\left\|I_{\lambda_{m}}(F) \phi\right\|^{2} \leqq M$ for all $m$. But $I_{\lambda_{m}}\left(F^{\prime}\right) \phi$ converges pointwise to $K_{-1}(F) \phi$ as $m \rightarrow \infty$.

Hence

$$
\begin{aligned}
\left\|K_{-1}(F) \phi\right\|^{2} & =\int_{-\infty}^{\infty}\left|\left(K_{-1}(F) \phi\right)(\xi)\right|^{2} d \xi \\
& \leqq \liminf \int_{-\infty}^{\infty}\left|\left(I_{\lambda_{m}}(F) \phi\right)(\xi)\right|^{2} d \xi \\
& =\lim \inf \left\|I_{\lambda_{m}}(F) \phi\right\|^{2} \\
& \leqq M
\end{aligned}
$$


which contradicts the fact that $K_{-1}(F) \phi \notin L_{2}$. Hence $I_{\lambda}(F) \phi$ doesn't have a weak $L_{2}$-limit as $\lambda \rightarrow i$ and so $J_{-1}(F)$ doesn't exist.

2. Analytic functions of finite sums of factorable finite-dimensional functionals.

For $1 \leqq j \leqq m$, let $a=t_{0, j}<\cdots<t_{n_{j}, j}=b$ be a partition of $[a, b]$. Also for $1 \leqq j \leqq m$ and $1 \leqq i \leqq n_{j}$, let $f_{i j}$ be a function of one variable. Finally, let $G$ be a function analytic at 0 . In this section we consider the existence of $J_{q}(F)$ where $F$ has the form $F(x)=G\left(\sum_{j=1}^{m} \prod_{i=1}^{n_{j}} f_{i j}\left[x\left(t_{i, j}\right)\right]\right)$. By multiplying, if necessary, by additional functions $f_{i j}$ which are identically 1 , we can, and will, assume that the partitions are the same for every $j$.

THEOREM 3. Let $a=t_{0}<t_{1}<\cdots<t_{n}=b$ be a partition of $[a, b]$. For each $1 \leqq j \leqq m$ and $1 \leqq i \leqq n$, let $f_{i j}$ be a function which is essentially bounded, say by $M_{i j}$. Suppose $G(z)=\sum_{k=0}^{\infty} a_{k} z^{k}$ is a function analytic at 0 with a radius of convergence $R>\sum_{j_{1=1}^{m}} \prod_{i=1}^{n} M_{i j} \equiv$ $M$. Let $F(x)=G\left(H_{0}(x)\right)$ where $H_{0}(x)=L\left(x\left(t_{1}\right), \cdots, x\left(t_{n}\right)\right)$ and, in turn, $L\left(u_{1}, \cdots, u_{n}\right)=\sum_{j=1}^{m} \prod_{i=1}^{n} f_{i j}\left(u_{i}\right)$. Then $J_{q}(F)$ exists for every $q \neq 0$ and, in fact, $I_{\lambda}(F) \rightarrow J_{q}(F)$ in the strong operator topology as $\lambda \rightarrow-i q$ in $C^{+}$. Moreover $\left\|I_{\lambda}(F)\right\| \leqq \sum_{k=0}^{\infty}\left|a_{k}\right| M^{k}$ for all $\lambda \in C^{+}$and $\left\|J_{q}(F)\right\| \leqq$ $\sum_{k=0}^{\infty}\left|a_{k}\right| M^{k}$ for every $q \neq 0$.

Proof. For each integer $k \geqq 0$, let $F_{k}(x)=\left[H_{0}(x)\right]^{k}$. From [4, pp. 777-8], one sees that $I_{\lambda}\left(F_{k}\right)$ and $J_{q}\left(F_{k}\right)$ exist for all $\lambda \in C^{+}$and all $q \neq 0$ respectively. Also by comparing $\left[H_{0}(x)\right]^{k}$ and $\left[\sum_{j=1}^{m} \prod_{i=1}^{n} M_{i j}\right]^{k}=$ $M^{k}$ and using the decomposition of $I_{\lambda}\left(F_{k}\right)$ and $J_{q}\left(F_{k}\right)$ described in [4, p. 778], one sees that $\left\|I_{\lambda}\left(F_{k}\right)\right\| \leqq M^{k}$ and $\left\|J_{q}\left(F_{k}\right)\right\| \leqq M^{k}$. Further $I_{2}\left(F_{k}\right)$ is given by

$$
\begin{aligned}
\left(I_{\lambda}\left(F_{k}\right) \psi\right)(\xi)= & \lambda^{n / 2} A \int_{-\infty}^{\infty}(n) \int_{-\infty}^{\infty} \psi\left(u_{n}\right) L^{k}\left(u_{1}, \cdots, u_{n}\right) \\
& \times \exp \left(-\sum_{j=1}^{n} \frac{\lambda\left(u_{j}-u_{j-1}\right)^{2}}{2\left(t_{j}-t_{j-1}\right)}\right) d u_{1} \cdots d u_{n} .
\end{aligned}
$$

$J_{q}\left(F_{k}\right)$ is given by a similar formula but with $\lambda$ replaced by $-q i$ and the integrals interpreted in the mean.

The existence of $I_{\lambda}(F)$ for $\lambda \in C^{+}$follows from Theorem 1 and we have

$$
\begin{aligned}
\left(I_{\lambda}(F) \psi\right)(\xi)= & \lambda^{n / 2} A \int_{-\infty}^{\infty}(n) \int \psi\left(u_{n}\right)\left[\sum_{k=0}^{\infty} a_{k} L^{k}\left(u_{1}, \cdots, u_{n}\right)\right] \\
& \times \exp \left(-\sum_{j=1}^{n} \frac{\lambda\left(u_{j}-u_{j-1}\right)^{2}}{2\left(t_{j}-t_{j-1}\right)}\right) d u_{1} \cdots d u_{n} .
\end{aligned}
$$

Now since $\left\|\sum_{k=n_{1}}^{n_{2}} a_{k} I_{\lambda}\left(F_{k}\right) \psi\right\| \leqq \sum_{k=n_{1}}^{n_{2}}\left|a_{k}\right| M^{k}$, it is clear that for every 
$\psi \in L_{2}, I_{\lambda}^{N}(F) \psi \equiv \sum_{k=0}^{N} a_{k} I_{\lambda}\left(F_{k}\right) \psi$ converges in $L_{2}$-norm as $N \rightarrow \infty$. But taking

$$
|\lambda|^{n / 2} A\left|\psi\left(u_{n}\right)\right|\left[\sum_{k=0}^{\infty}\left|a_{k}\right| M^{k}\right] \exp \left[-\sum_{j=1}^{n} \frac{\operatorname{Re} \lambda\left(u_{j}-u_{j-1}\right)^{2}}{2\left(t_{j}-t_{j-1}\right)}\right]
$$

as a dominating function and applying the Dominated Convergence Theorem much as in the proof of Theorem 1, we see that $I_{\lambda}^{N}(F) \psi$ converges pointwise to $I_{2}(F) \psi$. Hence we must have

$$
\left\|I_{\lambda}\left(F^{\prime}\right) \psi-I_{\lambda}^{N}(F) \psi\right\| \rightarrow 0 \text { as } N \rightarrow \infty \text {. }
$$

Now let $K_{q}(F) \equiv \sum_{k=0}^{\infty} J_{q}\left(F_{k}\right)$ and $J_{q}^{N}(F) \psi=\sum_{k=0}^{N} J_{q}\left(F_{k}\right) \psi$. From our earlier estimates on $\left\|J_{q}\left(F_{k}\right)\right\|$, it is clear that this makes sense and

$$
\left\|K_{q}(F) \psi-J_{q}^{N}(F) \psi\right\| \rightarrow 0 \quad \text { as } \quad N \rightarrow \infty .
$$

We finish the proof by showing that $I_{\lambda}(F) \rightarrow K_{q}(F)$ in the strong operator topology as $\lambda \rightarrow-i q$ in $C^{+}$. (Once this is done, the norm estimates on $J_{q}(F)$ and $I_{\lambda}(F)$ follow readily from (10) and (11).) But the desired convergence follows from (10) and (11) and the fact [4, p. 778] that $I_{i}^{N}(F) \rightarrow J_{q}^{N}(F)$ in the strong operator topology as $\lambda \rightarrow-i q$ in $C^{-}$.

We should remark that the present theorem is related to the theorem in [4] in much the same way that Theorems 7 and 5 of [2] are related.

\section{REFERENCES}

1. John A. Beekman and Ralph A. Kallman, Gaussian Markov expectations and related integral equations, Pacific J. Math., 37 (1971), 303-318.

2. R. H. Cameron and D. A. Storvick, An operator-valued function space integral and a related integral equation, J. Math. and Mech., 18 (1968), 517-552.

3. __ An integral equation related to the Schroedinger equation with an application to integration in function space, "Problems in analysis", Princeton Univ. Press, Princeton, New Jersey (1970), 175-193.

4. G. W. Johnson and D. L. Skoug, Operator-valued Feynman integrals of certain finite-dimensional functionals, Proc. Amer. Math. Soc., 24 (1970) 774-780.

5. An operator valued function space integral: A sequel to Cameron and Storvick's paper, Proc. Amer. Math. Soc., 27 (1971), 514-519.

6.- Operator-valued Feynman integrals of finite-dimensional functionals, Pacific J. Math., 34 (1970), 415-425.

Received July 8, 1970 and in revised form October 27, 1972. The authors would like to thank Professor R. H. Cameron who suggested to us that some extra hypotheses which appeared in an earlier version of Theorem 2 were unnecessary. The first author wishes to thank the University of Nebraska, Research Council for financial support. 



\section{PACIFIC JOURNAL OF MATHEMATICS}

EDITORS

\author{
H. SAMELSON \\ Stanford University \\ Stanford, California 94305 \\ C. R. HOBBY \\ University of Washington \\ Seattle, Washington 98105
}

\author{
J. DUGUNDJI \\ Department of Mathematics \\ University of Southern California \\ Los Angeles, California 90007 \\ RICHARD ARENS \\ University of California \\ Los Angeles, California 90024
}

\section{ASSOCIATE EDITORS}

E. F. BECKENBACH

B. H. NeUManN

F. WOLF

K. YosHIDA

\section{SUPPORTING INSTITUTIONS}

UNIVERSITY OF BRITISH COLUMBIA

CALIFORNIA INSTITUTE OF TECHNOLOGY

UNIVERSITY OF CALIFORNIA

MONTANA STATE UNIVERSITY

UNIVERSITY OF NEVADA

NEW MEXICO STATE UNIVERSITY

OREGON STATE UNIVERSITY

UNIVERSITY OF OREGON

OSAKA UNIVERSITY
UNIVERSITY OF SOUTHERN CALIFORNIA

STANFORD UNIVERSITY

UNIVERSITY OF TOKYO

UNIVERSITY OF UTAH

WASHINGTON STATE UNIVERSITY UNIVERSITY OF WASHINGTON

AMERICAN MATHEMATICAL SOCIETY NAVAL WEAPONS CENTER 


\section{Pacific Journal of Mathematics}

\section{Vol. 45, No. $1 \quad$ September, 1973}

William George Bade, Complementation problems for the Baire classes .......... 1

Ian Douglas Brown, Representation of finitely generated nilpotent groups ........ 13

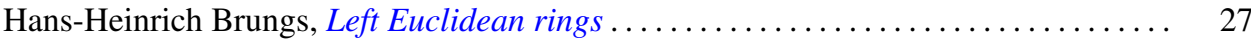

Victor P. Camillo and John Cozzens, A theorem on Noetherian hereditary rings ..... 35

James Cecil Cantrell, Codimension one embeddings of manifolds with locally flat

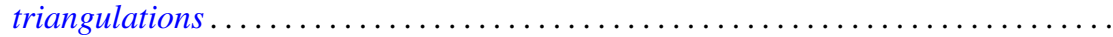

L. Carlitz, Enumeration of up-down permutations by number of rises . . . . . . . . . .

Thomas Ashland Chapman, Surgery and handle straightening in Hilbert cube

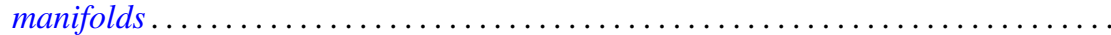

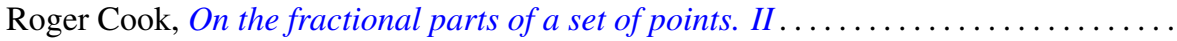

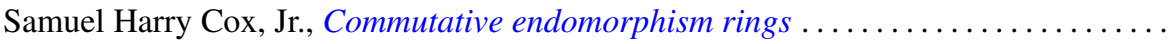

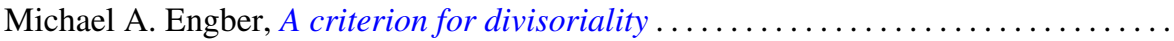

Carl Clifton Faith, When are proper cyclics injective . . . . . . . . . . . . . . 97

David Finkel, Local control and factorization of the focal subgroup . . . . . . . . . 113

Theodore William Gamelin and John Brady Garnett, Bounded approximation by

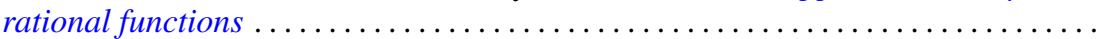

Kazimierz Goebel, On the minimal displacement of points under Lipschitzian

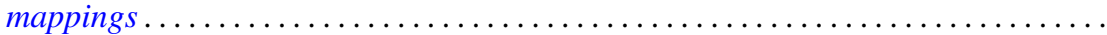

Frederick Paul Greenleaf and Martin Allen Moskowitz, Cyclic vectors for representations associated with positive definite measures: nonseparable

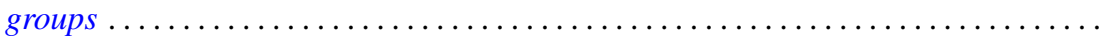

Thomas Guy Hallam and Nelson Onuchic, Asymptotic relations between perturbed linear systems of ordinary differential equations .

David Kent Harrison and Hoyt D. Warner, Infinite primes of fields and completions. .

James Michael Hornell, Divisorial complete intersections . ......

Jan W. Jaworowski, Equivariant extensions of maps ..............

John Jobe, Dendrites, dimension, and the inverse arc function .. .

Gerald William Johnson and David Lee Skoug, Feynman integrals of non-factorable

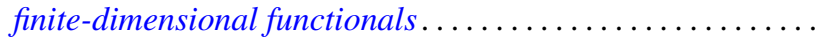

Dong S. Kim, A boundary for the algebras of bounded holomorphic functions ...... 269

Abel Klein, Renormalized products of the generalized free field and its derivatives ... 275

Joseph Michael Lambert, Simultaneous approximation and interpolation in $L_{1}$ and

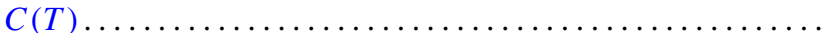

Kelly Denis McKennon, Multipliers of type $(p, p)$ and multipliers of the group

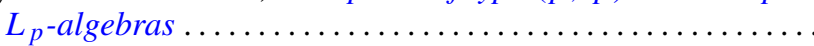

William Charles Nemitz and Thomas Paul Whaley, Varieties of implicative

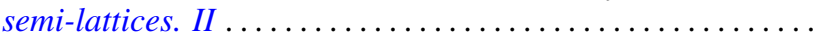

Donald Steven Passman, Some isolated subsets of infinite solvable

Norma Mary Piacun and Li Pi Su, Wallman compactifications on E-completely

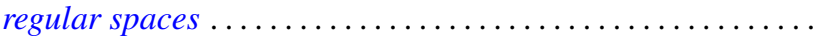

Jack Ray Porter and Charles I. Votaw, $S(\alpha)$ spaces and regular Hausdorff extensions....

Gary Sampson, Two-sided $L_{p}$ estimates of convolution transforms .

Ralph Edwin Showalter, Equations with operators forming a rig
Raymond Earl Smithson, Fixed points in partially ordered sets .

Victor Snaith and John James Ucci, Three remarks on symmetric products and

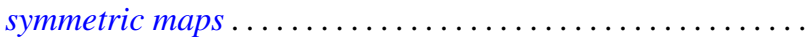

